\title{
A Review of Hyperbaric Oxygen Therapy for Inflammatory Bowel Disease
}

\author{
Naif Alenazi \\ Hesham Alsaeed' \\ Adel Alsulami ${ }^{1}$ \\ Turki Alanzi $\mathbb{D D}^{2}$ \\ 'Diving and Hyperbaric Medicine \\ Department, Diving Unit, Medical \\ Services, Dammam, Saudi Arabia; \\ ${ }^{2}$ Department of Health Information \\ Management and Technology, College of \\ Public Health, Imam Abdulrahman Bin \\ Faisal University, Dammam, Saudi Arabia
}

Correspondence: Turki Alanzi Department of Health Information Management and Technology, College of Public Health, Imam Abdulrahman Bin Faisal University, King Faisal Road 12,

Dammam, 3|44I, Saudi Arabia

Tel +966 I3333।2II

Email talanzi@iau.edu.sa

\begin{abstract}
IBD (inflammatory bowel disease) characterized by chronic inflammation of the gastrointestinal (GI) tract is one of the chronic diseases the prevalence of which has been increasing globally. In the past few decades, significant improvements were made in the treatments relating to IBD which have reduced the hospitalization and mortality rates; however, there is still room for improvement with other alternative therapies. HBOT (hyperbaric oxygen treatment) is one of the effective therapies used in treating various conditions including wounds, decompression sickness, stroke, etc. Several studies have reported the role of HBOT as an adjunctive treatment to IBD, while adopting conventional treatment procedures. There is an increasing trend of research in this particular area. Studies have shown that HBOT reflects its therapeutic effect by controlling inflammation, reducing oxidation stress, improving the process of cleaning damaged cells and recruiting the cells involved in repair; thereby improving the immunity response system. In this context, the purpose of this review is to summarize past clinical and experimental studies and to understand the role of hyperbaric treatment for IBD. The findings from the review have suggested that hyperbaric therapy can be an effective adjunctive approach for IBD, based on which some ideas for future clinical and research work are provided.
\end{abstract}

Keywords: hyperbaric oxygen therapy, inflammation, inflammatory bowel disease, chronic

\section{Introduction}

Inflammatory bowel disease (IBD) is a chronic condition, which is characterized by chronic inflammation of the gastrointestinal (GI) tract. IBDs mainly comprise Crohn's disease and ulcerative colitis, whose symptoms mainly include abdominal pain, rectal bleeding, diarrhea and fatigue. These conditions have a detrimental impact on patient quality of life and are associated with a high-cost burden, especially in the case of moderate to severe disease. ${ }^{1}$ The treatment for IBD focuses on maintaining long-term remission by healing the gut mucosa for delaying the progression of disease and related complications. ${ }^{2}$ While the exact causes of IBD are not known, it is linked to the defective immune system. ${ }^{3}$ The exact causes of IBD are not known, but the studies have identified various causes such as genetic factors and disruptions in immune response systems, etc. The common damage of both types of IBD results in inflammation of mucosa (inner lining of the intestinal tract), which may cause ulcers, bleeding, and electrolyte loss. ${ }^{4}$ Also, genetic factors such as deficits in autophagy (cleaning out damaged cells in order to regenerate newer and healthier cells) may play a role. ${ }^{5,6}$ Furthermore, in relation to disruptions in immune response, cytokines, which are responsible for communication between cells, bind to different receptors, which may alleviate the condition leading to 
chronic inflammatory process. ${ }^{7-9}$ Various types of treatment procedures including antibiotics, immunomodulators, and surgical intervention reflect a decrease in the rate of hospitalization. ${ }^{10,11}$

Furthermore, the prevalence of IBD has been rapidly increasing in the past few years. A recent study ${ }^{12}$ investigated the IBD cases from 1990 to 2017, and identified that the agestandardized global prevalence rate increased from 79.5 per 100,000 population in 1990 to 84.3 per 100,000 population in 2017. The US had the highest age-standardized prevalence rate of IBD of 464.5 per 100,000 population, followed by the UK with 449.6 per 100,000 population. Similarly, it was assessed that IBD prevalence is found to be higher in developed countries and urban regions, and lower in developing regions and rural areas. ${ }^{13}$ However, a systematic review of 147 studies related to IBD found that the prevalence was higher in Europe and North America, and the prevalence rates in newly industrialized countries in Asia have been slowly rising, which is a cause for concern. ${ }^{16}$ Furthermore, over 700,000 physician visits and 100,000 hospitalization cases were related to IBD in 2012 in the US alone. ${ }^{5}$ As per 2015 estimates, $1.3 \%$ of US adults (accounting for 3 million people) were diagnosed with IBD in $2015 .^{14}$ The hospitalization rates of IBD in the US have increased significantly in the past few years, which has led to an increase in healthcare costs: an average hospitalization cost of $\$ 13,000$ per person, which reflects an annual increase of $4 \%$ in healthcare costs. ${ }^{15}$ Moreover, IBD is associated with various chronic and infectious conditions; preventive care is thus an essential aspect of lifelong disease management.

Most of the studies focused on treatment with medication, such as salicylates, corticosteroids, immunosuppressants and immunomodulators, nutritional interventions, leukocytapheresis, etc. ${ }^{17-20}$ Procedures such as oxygen therapy can have various advantages such as increasing immunity, detoxing blood, stimulating brain activity, and enhancing recovery from inflammation and wounds. ${ }^{21-23}$ However, there is a lack of research on analyzing the role of the hyperbaric treatment procedure, which aims to increase oxygen supply to the damaged areas (intestinal ulcers, inflammation) for quick recovery. ${ }^{24}$ Considering this gap, this study focuses on reviewing the role of hyperbaric treatment for IBD, the complications associated with it, and possible areas for future research.

\section{Methods}

There are two standard types of review identified in research studies: systematic reviews and narrative reviews.
It is identified that, in medical literature, the number of narrative articles has significantly surpassed that of systematic reviews. ${ }^{25}$ The purpose of narrative reviews is to identify studies that describe a problem of interest and discuss it; there are no predetermined research questions, search strategies or protocols to guide the review. ${ }^{26}$ A narrative review may be organized in different formats: in a chronological order, with a summary of the history of research when clear trends are identified, or presented as a "conceptual framework", where the contents are separated according to dependent or independent variables and their relationships, or discussing the concepts related to a particular topic of interest. ${ }^{27}$ As this paper focuses on investigating the role of hyperbaric treatment for IBD, where there is a need to identify the articles relating to the treatment procedures, types of IBD, and the impact of procedures, there is a need to select and analyze papers in different contexts and, at the same time, to link the hyperbaric treatment procedures and IBD types. For this purpose, a narrative review method was adopted to review the relevant papers and discuss their findings in relation to the topic of interest, which is to review the role of hyperbaric treatment for IBD. Accordingly, the next part of the paper focuses on reviewing the different types of IBD, presents an overview of hyperbaric treatment procedures and, finally, discusses the applicability of the treatment procedures for IBD.

\section{Hyperbaric Oxygen Treatment (HBOT)}

IBD treatments mainly focuse on reducing the inflammation in the damaged area, thereby accelerating the healing process. Hyperbaric treatment is one such approach, which focuses on increasing the oxygen supply in the blood to accelerate the healing process. The hyperbaric treatment procedure has a long history, since its first use by Junod in 1834 in France to treat pulmonary conditions. ${ }^{28}$ In the early 20 th century, HBOT was used to treat a variety of conditions in Europe and the US. ${ }^{29}$ It is also used for radiation injuries, gas gangrene, carbon monoxide poisoning, and decompression sickness. ${ }^{29,30}$ HBOT is provided for patients in specialized chambers which are at 1.5 to 3 times higher than normal pressure at sea level. The chambers are filled with pure oxygen and aim to fill the blood with enough oxygen to repair tissues and improve the healing process. There are two types of chamber: a mono-place chamber (a long tube-like 
chamber into which a patient slips down while the chamber is slowly pressurized with $100 \%$ oxygen) and a multiplace chamber (a chamber or room that can fit two or more people, which has pressure greater than atmospheric pressure, where the patients breathe pure oxygen through masks). ${ }^{31}$ There are also several portable size chambers that can be used at home for treatment. These portable chambers are referred to as mild personal hyperbaric chambers, which have low pressure compared to hard chambers that are used in hospitals and clinics. In the US, these mild chambers are categorized as Class II medical devices which require a prescription to purchase. ${ }^{32}$ HBOT increases the oxygen supply to the damaged tissues, which can accelerate the healing process. Usually injuries damage the blood vessels, which in turn release fluids that leak into tissues, which leads to swelling. Swelling disrupts the oxygen supply to the damaged cells and tissues, as a result of which they start to die. In this context, HBOT increases the blood supply to the tissues and reduces the swelling in a pressurized chamber. In addition, hyperbaric treatment boosts the immune system by disabling the toxins produced by certain types of bacteria by increasing the oxygen supply to the tissues, which helps them to resist the infection. Also, the hyperbaric therapy improves the ability of white blood cells to identify the invaders and destroy them in the body. Furthermore, hyperbaric therapy stimulates cells to produce certain substances such as vascular endothelial growth factor, which attract and stimulate endothelial cells leading to the formation of new connective tissues and also new skin cells. ${ }^{31}$ However, there are certain potential risks associated with the treatment, which include: middle ear injuries, eardrum injuries, fluid and blood leakage, temporary myopia, lung collapse, etc. due to changes in air pressure; seizures in the central nervous system due to increased oxygen levels or oxygen toxicity; and lowering of sugar levels in diabetic patients treated with insulin. ${ }^{33-35}$ One of the major drawbacks of the treatment is high costs. Hyperbaric therapy is used for treating various conditions in different countries, and the price for the treatment varies across the countries. While few countries such as the UK provide the treatment for free through National Health Service outlets, in the US, the treatment is covered in Medicare, whereas in Australia, it is not covered in Medicare insurance scheme. For effective treatment results, there is a need for $20-40$ sessions of therapy, and one session usually costs $\$ 300$ in private clinics and about $\$ 2000$ in hospitals. ${ }^{36-38}$
Therefore, the treatment may not be affordable for all and not for all conditions, which is one of the major drawbacks.

\section{HBOT for Inflammatory Bowel Disease (IBD)}

HBOT has been used to treat a variety of diseases, including decompression sickness and healing of problem wounds, etc. Studies have analyzed the use of HBOT in treating IBD and various results have been identified. As IBD is associated with immune response malfunctioning, HBOT can be used to improve immunity by increasing the oxygen supply to the cells and tissues. Focusing on this aspect, a systematic review ${ }^{39}$ of 19 studies (13 related to Crohn's disease and six related to ulcerative colitis) analyzing the impact of HBOT on IBD were analyzed. Findings have shown that participants in all studies were treated with corticosteroids, immunomodulators and antiinflammatory medications. About $78 \%$ of patients (31/40) with Crohn's disease in 13 studies showed improvement with HBOT, and all patients (39/39) with ulcerative colitis in six studies showed improvement, indicating the positive and significant impact of HBOT on both types of IBD. Furthermore, decrease in pro-inflammatory cytokines (IL1, IL-6 and TNF-alpha), and markers of inflammation or immune dysregulation including TNF-alpha, IL-1beta, neopterin and myeloperoxidase activity was observed among the patients after HBOT. This systematic review ${ }^{39}$ has identified that HBOT can lower the markers of inflammation and oxidative stress, and ameliorate IBD. Similarly, effect of HBOT on the production of proinflammatory cytokines, nitric oxide and hypoxiainducible factor 1alpha was studied in vitro, ${ }^{40}$ which has identified that HBOT significantly prevented the proinflammatory cytokine production; affected myeloperoxidase activity, in the expression of inducible nitric oxide synthase and cyclooxygenase-2; and attenuated the severity of acute distal colitis. Another study ${ }^{41}$ investigating the safety and efficacy of HBOT for IBD reviewed 17 studies including 613 patients, out of which 286 were diagnosed with Crohn's disease and 327 with ulcerative colitis. Analyzing the 8924 treatments, nine adverse events were identified, of which six were serious; and the rate of adverse events when using HBOT for IBD was lower than using BOT for other indications. Therefore, HBOT is not only effective for treating IBDs but also safe. 
As IBD is associated with the production of reactive species that target cysteine redox switches in proteins, and intermediary metabolism, it has been identified in the research $^{42}$ that HBOT can reduce oxidative stress, thereby reducing the impact of IBD. HBOT was also proved to be effective in treating the other conditions caused by IBD treatments, such as proctectomy (removal of complete or part of rectum). Persistent perineal sinus (wound remaining unhealed more than 6 months after abdominoperineal resection) after proctectomy for IBD affected more than $50 \%$ of the patients; and it remained unhealed for two years in $33 \%$ of the cases despite optimal conventional therapy. ${ }^{43}$ To assess the impact of HBOT on these cases, a study ${ }^{43}$ has investigated four patients with extreme persistent perineal sinus who were given preoperative and postoperative HBOT treatment, and identified that complete healing was observed in all of them.

HBOT is also identified to be effective on both types of IBD including Crohn's disease and ulcerative colitis. A study ${ }^{44}$ has investigated the impact of HBOT on a patient with extremely debilitating complication of Crohn's disease which has not been cured for eight years. The disease worsened even after surgery and medical treatment (sulfasalazine, steroids, 6-mercaptopurine, metronidazole, antibiotics). HBOT was instituted while the patient was continued on metronidazole. After four courses of the therapy, the patient has become asymptomatic and the wounds are cured to a greater extent compared to the condition before the therapy. Similarly, HBOT was also identified to be effective in treating ulcerative colitis, as $100 \%$ clinical remission was achieved in a study. ${ }^{45}$ Therefore, HBOT can be effective for both types of IBD.

HBOT was also considered as an adjuvant or complimentary therapy for treating IBD. A recent study ${ }^{46}$ has investigated the efficacy of hyperbaric therapy as an alternate therapy for treating patients with severe ulcerative colitis. A randomized controlled trial with 20 patients divided into two equal groups (intervention and control) was conducted. The patients in the intervention group received 10 sessions of HBOT (five times per week) for two weeks along with $400 \mathrm{mg}$ hydrocortisone and intravenous (IV) fluids, while the control group only received $400 \mathrm{mg}$ hydrocortisone and intravenous (IV) fluids. Inflammatory markers including ESR, CRP, faecal calprotectin, rectal bleeding score (RBS), stool frequency score (SFS) and Mayo endoscopic score (MES) were used to evaluate the therapeutic effect. At the end of the two-week study, a significant decrease in ESR, CRP, faecal calprotectin, RBS, SFS and MES levels were identified in the intervention group compared to the control group. As HBOT can increase neovascularization, inhibition of bacterial growth, improved intestinal mucosal healing and increased intestinal motility, by improving the supply of oxygen to the tissues, it can be used as complementary and alternative therapies for IBD and other gastrointestinal tract disorders. ${ }^{47}$

HBOT was identified as an adjunctive therapy for treating IBD, but not considered as the main treatment, as the results from various studies varied and bias was observed. In a study ${ }^{48}$ focusing on the effect of HBOT on complicated Crohn's disease (in the patients who were refractory to conventional treatment practices), it was identified that HBOT was effective on patients with various complications including perianal fistulizing Crohn's disease (pCD) (80\% healing rate), enterocutaneous fistulas (ECF) $(84.6 \%)$, or pyoderma gangrenosum (PG) $(100 \%)$. Similarly, HBOT was also identified to be an effective adjunctive approach for treating metastatic Crohn's disease. ${ }^{49}$ Furthermore, HBOT treatment can result in achieving clinical remission within a week of starting the treatment and can restrict the progression to second-line therapy during the hospitalization. ${ }^{50}$

Focusing on the efficacy, safety, and feasibility of HBOT, studies have shown varying results. A recent study $^{51}$ investigated the efficacy, safety, and feasibility of HBOT in IBD patients with rectovaginal fistulas. Nine patients who had unsuccessful medical and/or surgical treatments for their RVF were treated with HBOT for three months and no patient showed any improvement in their condition. Similarly, another recent study ${ }^{52}$ identified that HBOT was effective in treating chronic antibioticrefractory pouchitis and other gastrointestinal inflammatory conditions. A significant reduction in the mean mPDAI symptom sub-score from 3.19 to 1.91 after HBOT $(\mathrm{p}<0.05)$ was observed among the 46 patients included in the study. Therefore, focusing on the efficacy, safety, and feasibility, it can be assessed that there are conflicting results among the various studies. Limited high-quality evidence suggests that HBOT is considered to be safe; however, the need for extensive research in this area is recognized. ${ }^{53,54}$ There are other benefits of HBOT for IBD, as it is identified to reduce the risk of rehospitalization, and mortality by over $30 \%$. In addition, it was identified that though HBOT costs more, it also yields higher quality-adjusted life years (QALYs), reflecting that hyperbaric therapy is more cost-effective than the standard of care. ${ }^{52}$ However, a recent systematic review ${ }^{55}$ 
including 18 studies identified that clinical remission was identified in $83.24 \%$ of patients with ulcerative colitis and $81.89 \%$ of patients with Crohn's disease from all studies, with no major adverse events identified; indicating the safety, reliability and efficacy of the hyperbaric treatment for IBD.

\section{Conclusion}

Various studies were reviewed to identify the various types of IBD and other relevant conditions. The review has identified that there is a lack of research related to the analysis of efficacy, safety, reliability, and costeffectiveness of hyperbaric treatment for different types of IBD conditions (IBD along with other related conditions). However, there are studies which have identified the impact of HBOT on tissue damage such as inflammation of mucosa. Clinical, experimental, and systematic review studies analyzed in this study have identified that HBOT reflects its therapeutic effect by controlling inflammation, reducing oxidation stress, improving the process of cleaning damaged cells and recruiting the cells involved in repair; thereby improving the immunity response system. Overall, hyperbaric treatment can be considered as an effective adjuvant approach for treating IBD; however, there is a need for further research in future in relation to the analysis of the impact on IBD with other relevant risk factors or conditions. Future studies can also focus on analyzing the impact of hyperbaric treatment for injuries by risk factors associated with IBD. Furthermore, hyperbaric treatment may be integrated with eHealth technology, for remotely monitoring the patients using mild hyperbaric chambers at home, which can also guide the future research. Finally, for the patients with IBD, the specific mechanisms underlying the therapeutic effect of HBOT are still unclear due to the lack of experimental studies; as a result, future studies can focus on well-defined experimental studies.

\section{Disclosure}

The authors report no conflicts of interest in this work.

\section{References}

1. Gomollón F, Dignass A, Annese V, et al. 3rd European evidence-based consensus on the diagnosis and management of Crohn's disease 2016: part 1: diagnosis and medical management. J Crohns Colitis. 2017;11 (1):3-25. doi:10.1093/ecco-jcc/jjw168
2. Brady JE, Stott-Miller M, Mu G, Perera S. Treatment patterns and sequencing in patients with inflammatory bowel disease. Clin Ther. 2018;40(9):1509-1521.e5. doi:10.1016/j.clinthera.2018.07.013

3. Center for Disease Control and Prevention. CDC -what is inflammatory bowel disease (IBD)? - inflammatory bowel disease - division of population health. Cdc.gov; 2021. Available from: https://www.cdc. gov/ibd/what-is-IBD.htm. Accessed October 15, 2021.

4. Thoreson R, Cullen JJ. Pathophysiology of inflammatory bowel disease: an overview. Surg Clin North Am. 2007;87(3):575-585. doi:10.1016/j.suc.2007.03.001

5. Rioux JD, Xavier RJ, Taylor KD, et al. Genome-wide association study identifies new susceptibility loci for Crohn disease and implicates autophagy in disease pathogenesis. Nat Genet. 2007;39 (5):596-604. doi:10.1038/ng2032

6. Podolsky D. Inflammatory Bowel Disease. N Engl J Med. 1991;325 (13):928-937. doi:10.1056/NEJM199109263251306

7. Sianos EV, Katsanos KH, Tsianos VE. Role of genetics in the diagnosis and prognosis of Crohn's disease. World J Gastroenterol. 2012;18(2):105-118. doi:10.3748/wjg.v18.i2.105

8. Lashner B. Inflammatory bowel disease. In: Carey WD, editor. Cleveland Clininc: Current Clinical Medicine. Philadelphia, PA: Saunders; 2009.

9. Lennard-Jones J. Classification of inflammatory bowel disease. Scand $J$ Gastroenterol. 1989;24(sup170):2-6. doi:10.3109/00365528 909091339

10. Lichtenstein GR, Abreu MT, Cohen R, Tremaine W. American Gastroenterological Association Institute medical position statement on corticosteroids, immunomodulators, and infliximab in inflammatory bowel disease. Gastroenterology. 2006;130(3):935-939. doi:10.1053/j.gastro.2006.01.047

11. Feagan BG, Lémann M, Befrits R, et al. Recommendations for the treatment of Crohn's disease with tumor necrosis factor antagonists: an expert consensus report. Inflamm Bowel Dis. 2012;18(1):152-160. doi:10.1002/ibd.21870

12. Alatab S, Sepanlou S, Ikuta K, et al. The global, regional, and national burden of inflammatory bowel disease in 195 countries and territories, 1990-2017: a systematic analysis for the Global Burden of Disease Study 2017. Lancet Gastroenterol Hepatol. 2020;5(1):17-30. doi:10.1016/S2468-1253(19)30333-4

13. William R, Lichtenstein GR, Anand BS et al. Inflammatory bowel disease: epidemiology; 2021. Available from: https://www.cdc.gov/ ibd/data-statistics.htm\#6. Accessed October 15, 2021.

14. Center for Disease Control and Prevention. Data and statisticsinflammatory bowel. Cdc.gov; 2021. Available from: https://www. cdc.gov/ibd/what-is-IBD.htm. Accessed October 15, 2021.

15. Xu F, Liu Y, Wheaton AG, Rabarison KM, Croft JB. Trends and factors associated with hospitalization costs for inflammatory bowel disease in the United States. Appl Health Econ Health Policy. 2019;17(1):77-91. doi:10.1007/s40258-018-0432-4

16. Ng SC, Shi HY, Hamidi N, et al. Worldwide incidence and prevalence of inflammatory bowel disease in the 21 st century: a systematic review of population-based studies [published correction appears in Lancet. $\quad 2020$ Oct 3;396(10256):e56]. Lancet. 2017;390 (10114):2769-2778. doi:10.1016/S0140-6736(17)32448-0

17. Engel MA, Neurath MF. New pathophysiological insights and modern treatment of IBD. $J$ Gastroenterol. 2010;45:571-583. doi:10.1007/s00535-010-0219-3

18. Sigurbjörnsson F, Bjarnason I. Leukocytapheresis for the treatment of IBD. Nat Rev Gastroenterol Hepatol. 2008;5:509-516. doi:10.1038/ ncpgasthep1209

19. Knox NC, Forbes JD, Van Domselaar G, et al. The gut microbiome as a target for IBD treatment: are we there yet? Curr Treat Options Gastro. 2019;17:115-126. doi:10.1007/s11938-019-00221-w

20. Pigneur B, Ruemmele F. Nutritional interventions for the treatment of IBD: current evidence and controversies. Therap Adv Gastroenterol. 2019;12:175628481989053. doi:10.1177/1756284819890534 
21. Lindsey R. Topic: oxygen is essential for brain activity; 2020. Available from: https://valeowc.com/the-benefits-of-oxygen/. Accessed October 15, 2021.

22. Bennett MH, Trytko B, Jonker B. Hyperbaric oxygen therapy for the adjunctive treatment of traumatic brain injury. Cochrane Database Syst Rev. 2012;12:CD004609.

23. Lansdorp CA, Gecse KB, Buskens CJ, et al. Hyperbaric oxygen therapy for the treatment of perianal fistulas in Crohn's disease (HOT-TOPIC): study protocol of a prospective interventional cohort study with one-year follow-up. Undersea Hyperb Med. 2019;46 (1):45-53. doi:10.22462/01.03.2019.5

24. Bastian H, Glasziou P, Chalmers I. Seventy-five trials and eleven systematic reviews a day: how will we ever keep up? PLoS Med. 2010;7:e1000326. doi:10.1371/journal.pmed.1000326

25. Demiris G, Oliver D, Washington K. Defining and analyzing the problem. Behav Interv Res Hospice Palliat Care. 2019;15:27-39.

26. Ferrari R. Writing narrative style literature reviews. Eur Med Writers Assoc. 2015;24(4):230-236.

27. Mayo Clinic. Inflammatory bowel disease (IBD) - symptoms and causes. Mayo Clinic; 2021. Available from: https://www.mayocli nic.org/diseases-conditions/inflammatory-bowel-disease/symptomscauses/syc-20353315. Accessed October 15, 2021.

28. Encyclopaedia Britannica Inc. Aerotherapeutics. 11th ed. Encyclopaedia Britannica Inc; 1911:271.

29. Sharkey S. Current indications for hyperbaric oxygen therapy Lieutenant. ADF Health. 2001;1(2):64-72.

30. Brummelkamp WH, Hogendijk L, Boerema I. Treatment of anaerobic infections (clostridial myositis) by drenching the tissues with oxygen under high atmospheric pressure. Surgery. 1961;49:299-302.

31. Johns Hopkins Medicine. Hyperbaric oxygen therapy; 2021. Available from: https://www.hopkinsmedicine.org/health/treatmenttests-and-therapies/hyperbaric-oxygen-therapy. Accessed October $15,2021$.

32. US Food \& Drug. "Product classification, chamber, hyperbaric"; 2021. Available from: https://www.accessdata.fda.gov/scripts/cdrh/ cfdocs $/ \mathrm{cfPCD} /$ classification.cfm?ID=CBF. Accessed October 15, 2021.

33. Mayo Clinic. Topic hyperbaric oxygen treatment; 2021. Available from: https://www.mayoclinic.org/tests-procedures/hyperbaricoxygen-therapy/about/pac-20394380. Accessed October 15, 2021.

34. Butler FK. Diving and hyperbaric ophthalmology. Survey Opthamol. 1995;39(5):347-366. doi:10.1016/S0039-6257(05)80091-8

35. Smerz RW. Incidence of oxygen toxicity during the treatment of dysbarism. Undersea Hyperbaric Med Soc Inc. 2004;31(2):199-202.

36. Jain KK. Effect of hyperbaric oxygenation on spasticity in stroke patients. J Hyperbaric Med. 1989;4(2):55-61.

37. Singhal A, Lo E. Advances in emerging nondrug therapies for acute stroke 2007. Stroke. 2008;39(2):289-291. doi:10.1161/STROKE AHA.107.511485

38. Jain K. Textbook of Hyperbaric Medicine. 6th ed. Springer; 2017.

39. Rossignol DA. Hyperbaric oxygen treatment for inflammatory bowel disease: a systematic review and analysis. Med Gas Res. 2012;2:6. doi:10.1186/2045-9912-2-6

40. Parra R, Lopes A, Carreira E, et al. Hyperbaric oxygen therapy ameliorates TNBS-induced acute distal colitis in rats. Med Gas Res. 2015;5(1). doi:10.1186/s13618-015-0026-2.
41. Dulai PS, Gleeson MW, Taylor D, Holubar SD, Buckey JC, Siegel CA. Systematic review: the safety and efficacy of hyperbaric oxygen therapy for inflammatory bowel disease. Aliment Pharmacol Ther. 2014;39(11):1266-1275. doi:10.1111/apt.12753

42. Bourgonje A, Feelisch M, Faber K, Pasch A, Dijkstra G, van Goor H. Oxidative stress and redox-modulating therapeutics in inflammatory bowel disease. Trends Mol Med. 2020;26(11):1034-1046. doi:10.1016/j.molmed.2020.06.006

43. Chan X, Koh C, Glover M, Bryson P, Travis S, Mortensen N. Healing under pressure: hyperbaric oxygen and myocutaneous flap repair for extreme persistent perineal sinus after proctectomy for inflammatory bowel disease. Colorect Dis. 2014;16(3):186-190. doi:10.1111/ codi. 12500

44. Brady C, Cooley B, Davis J. Healing of severe perineal and cutaneous Crohn's disease with hyperbaric oxygen. Gastroenterology. 1989;97(3):756-760. doi:10.1016/0016-5085(89)90649-5

45. Buchman AL, Fife C, Torres C, Smith L, Aristizibal J. Hyperbaric oxygen therapy for severe ulcerative colitis. J Clin Gastroenterol. 2001;33(4):337-339. doi:10.1097/00004836-200110000-00018

46. Abdel-Hakim MM, Elsakaty TM, Kaisar HH, Shabana SS, Mohamed MA. Role of hyperbaric oxygen as an adjuvant therapy in severe ulcerative colitis patients. Ann RSCB. 2021;25:7998-8009.

47. Ismail A. Hyperbaric oxygen therapy as a complementary or alternative therapy for chronic oral and gastrointestinal disorders: a narrative review. Int J Alt Comp Med. 2020;1:33.

48. Feitosa MR, Parra RS, Machado VF, et al. Adjunctive hyperbaric oxygen therapy in refractory Crohn's disease: an observational study. Gastroenterol Res Pract. 2021;2021:6628142. doi:10.1155/2021/ 6628142

49. Lansdorp CA, Buskens CJ, Gecse KB, D’Haens GR, Van Hulst RA. Wound healing of metastatic perineal Crohn's disease using hyperbaric oxygen therapy: a case series. United Eur Gastroenterol J. 2020;8(7):820-827. doi:10.1177/2050640620934915

50. Dulai PS, Buckey JC, Raffals LE, et al. Hyperbaric oxygen therapy is well tolerated and effective for ulcerative colitis patients hospitalized for moderate-severe flares: a phase $2 \mathrm{~A}$ pilot multi-center, randomized, double-blind, sham-controlled trial. Am J Gastroenterol. 2018;113(10):1516-1523. doi:10.1038/s41395-018-0005-z

51. Lansdorp C, Buskens C, Gecse K, D'Haens G, van Hulst R. Hyperbaric oxygen therapy for the treatment of rectovaginal fistulas in patients with Crohn's disease: results of the HOT-REVA pilot study. BJS Open. 2021;5(3). doi:10.1093/bjsopen/zrab042

52. Hasan B, Yim Y, Ur Rashid M, et al. Hyperbaric oxygen therapy in chronic inflammatory conditions of the pouch. Inflamm Bowel Dis. 2020;27(7):965-970. doi:10.1093/ibd/izaa245

53. Dulai P, Jairath V. A microsimulation model to project the 5-year impact of using hyperbaric oxygen therapy for ulcerative colitis patients hospitalized for acute flares. Dig Dis Sci. 2020;66:3740-3752.

54. McCurdy J, Siw K, Kandel R, Larrigan S, Rosenfeld G, Boet S. The effectiveness and safety of hyperbaric oxygen therapy in various phenotypes of inflammatory bowel disease: systematic review with meta-analysis. Inflamm Bowel Dis. 2021. doi:10.1093/ibd/izab098

55. Singh A, Jha D, Jena A, Kumar MP, Sebastian S, Sharma V. Hyperbaric oxygen therapy in inflammatory bowel disease. Eur $J$ Gastroenterol Hepatol. 2021;33:Publish Ahead of Print. doi:10.1097/MEG.0000000000002164 


\section{Publish your work in this journal}

The International Journal of General Medicine is an international, peer-reviewed open-access journal that focuses on general and internal medicine, pathogenesis, epidemiology, diagnosis, monitoring and treatment protocols. The journal is characterized by the rapid reporting of reviews, original research and clinical studies across all disease areas. The manuscript management system is completely online and includes a very quick and fair peer-review system, which is all easy to use. Visit http://www.dovepress.com/ testimonials.php to read real quotes from published authors.

Submit your manuscript here: https://www.dovepress.com/international-journal-of-general-medicine-journal 